\title{
Assessment of Natural Deep Eutectic Solvent Pretreatment on Sugar Production from Lignocellulosic Biomass
}

\author{
Yoon Li Wan ${ }^{1 *}$, and Yuen Jun Mun ${ }^{1}$ \\ ${ }^{1}$ School of Engineering, Taylor's University, Subang Jaya, Selangor, Malaysia
}

\begin{abstract}
Before the conversion of lignocellulosic biomass into fuel such as ethanol, the biomass needs to be pretreated and the yield of ethanol is highly dependent on the pretreatment efficiency. This study investigate the performance of deep eutectic solvent (DES) in pretreating sago waste which is a type of starchy biomass. The suitable type of DES in sago waste pretreatment was selected based on three criteria, which is the structural characteristic, the sugar yield during enzymatic hydrolysis and the amount of sugar loss during pretreatment. In this study, three types of DES namely Choline Chloride-Urea (ChCl-Urea), Choline Chloride-Citric acid (ChCl-CA) and Choline Chloride-Glycerol (ChCl-Glycerol) was investigated. The effect of temperature and duration on DES pretreatment was also investigated. All DES reagents were able to disrupt the structure and increase the porosity of sago waste during pretreatment. $\mathrm{ChCl}$ Urea was selected in this study as it shows apparent structural disruption as examined under Scanning Electron Microscope (SEM). The highest glucose yield of $5.2 \mathrm{mg} / \mathrm{mL}$ was derived from enzymatic hydrolysis of ChCl-Urea pretreated sago waste. Moreover, reducing sugar loss during ChCl-Urea pretreatment was low, with only $0.8 \mathrm{mg} / \mathrm{mL}$ recorded. The most suitable temperature and duration for ChCl-Urea pretreatment is at $110^{\circ} \mathrm{C}$ and $3 \mathrm{hr}$. In a nutshell, the application of DES in pretreatment is feasible and other aspects such as the biodegradability and recyclability of DES is worth investigating to improve the economic feasibility of this pretreatment technique.
\end{abstract}

\section{Introduction}

Lignocellulosic biomass has the potential to replace existing petroleum-dependant energy source due to its abundant and renewable nature [1]. Many studies have shown that lignocellulosic biomass can be potentially converted to biofuel such as bioethanol and biodiesel [2]. In common practice, bioethanol is produced by following a pathway of biomass pretreatment, enzymatic hydrolysis, fermentation and distillation [3]. Pretreatment is the first process in lignocellulosic biomass conversion and hence, the efficiency of pretreatment process is the key to effective enzymatic hydrolysis and related downstream processes [3]. Pretreatment is necessary due to remove the lignin content in the biomass which resists solubilization and hamper enzymatic hydrolysis process [4]. In addition to that, factors such as cellulosic compositions, crystallinity structure, rigid nature of cellulose,

\footnotetext{
* Corresponding author: LiWan.Yoon@taylors.edu.my
} 
formation of inhibitors and particle size of biomass could contribute to the enzymatic digestibility of biomass [4].

Many approaches such as physical, chemical and biological pretreatment methods have been applied. Pretreated substrate would normally have a structure with increased porosity and reduced crystallinity [4]. During pretreatment, modification or removal of lignin content takes place to improve the accessibility of enzymes to the biomass substrate. The conversion of complex carbohydrates into simple sugars and subsequently fermented into bioethanol could be improved when pretreated substrate is applied [3]. However, as good as conventional pretreatments may be beneficial to sugar production from biomass, these methods have their respective drawback. For example, acid pretreatment produce fermentation inhibitors that could reduce the efficiency of fermentation.

The desire for green solvents in pretreatment has led to discovery of deep eutectic solvents (DES) and ionic liquids (IL) [5]. DES is made up of a hydrogen bond donor (HBD) and a hydrogen bond acceptor (HBA). Both DES and IL pretreatment techniques are affected by combination of chemicals used to produce the solvents, the type of biomass feedstock, size of particles and substrate loading [6 - 9]. These advanced pretreatment techniques are gaining attentions as extreme operating conditions such as high pressure and temperature is not required.

Further advancement of DES has been made due to its potential as green solvents to replace IL. Due to low costs and easily accessible raw materials, DES are being studied extensively in various fields such as electrochemistry and pharmaceutical [5]. DES has similar physio-chemical properties to IL with extra benefits such as easier preparation, noncomplicated purification processes and it is recyclable [9]. Majority of the DES are Choline Chloride-based $(\mathrm{ChCl})$, which is applied as food additive. With that being said, $\mathrm{ChCl}$ is acknowledged as safe compound for human consumption by relevant authorities [10]. Many researches have evaluated degradability of DES and have classified DESs as readily biodegradable compounds [9]. Combination of $\mathrm{ChCl}$ into DES has shown significant enhancement of biodegradability. Recently, the potential application of DES in pretreatment of biomass has been proven positive as the enhancement of sugar production from DES-pretreated biomass was observed in a few studies [7 - 9].

To date, no research has been done on the effect of DES pretreatment on starchy biomass. Starchy biomass such as sago waste which has approximately $36 \%$ starch, $40 \%$ cellulose and $13 \%$ hemicellulose serves as a promising feedstock for glucose production. In this study, three types of DES namely Choline Chloride-Urea, Choline Chloride-Citric acid and Choline Chloride-Glycerol was investigated for the pretreatment of sago waste. These DES were selected as they are commonly applied as pretreatment agents in other study [7 9]. The best DES was selected based on sugar production from enzymatic hydrolysis while investigating the sugar loss during pretreatment. Structural characteristic was also serves as one of the criteria for selection of DES. The effect of temperature and duration on DES pretreatment on sago waste was also investigated.

\section{Methodology}

\subsection{Preparation of Deep Eutectic Solvent (DES)}

Three types of DES, namely choline chloride with urea (ChCl-urea), citric acid (ChCl-CA) and glycerol (ChCl-Glycerol) were prepared at molar ratio of 1:2, 1:2 and 1:1 respectively [7 -9]. The mixture was heated by using hot plate with stirring rate of $150 \mathrm{rpm}$ at $80^{\circ} \mathrm{C}$. The heating and stirring process was halted when a homogenous and clear solution was 
obtained. The solution was cooled down before it was stored in a tightly-sealed bottle. The DES solution was kept and stored in a dessicator with silica gel.

\subsection{Preparation of Sago Waste}

Sago waste was collected from Hak Mong Sago Mill, Batu Pahat, Johor, Malaysia. The sample was washed and dried at $60^{\circ} \mathrm{C}$ for $24 \mathrm{hr}$. Sago waste was grinded and sieved to particle size of approximately $500 \mu \mathrm{m}$ prior to be used in pretreatment.

\subsection{DES pretreatment}

Pretreatment was carried out with $5 \%$ solid loading by mixing $1 \mathrm{~g}$ of sago waste and $20 \mathrm{~g}$ of DES respectively in a Schott bottle. The samples were heated at $110^{\circ} \mathrm{C}$ for 3 hours in the oven (UN-75, Memmert). The biomass/DES mixtures were then transferred to centrifuge tubes with the addition of $5 \mathrm{ml}$ deionized water. The contents were centrifuged at $3500 \mathrm{rpm}$ for approximately 30 minutes. The supernatant was collected into sampling bottle and stored at $4^{\circ} \mathrm{C}$. Reducing sugar concentration in the supernatant collected from pretreatment was subjected to DNS analysis. Washing and rinsing of biomass was then carried out by centrifugation. The pretreated biomass was then dried at $50^{\circ} \mathrm{C}$ and kept in desiccator before subjecting it to hydrolysis process and scanning electron microscopy (SEM) analysis.

\subsection{Enzymatic hydrolysis}

Enzymatic hydrolysis was conducted in $2 \%$ solid loading by mixing $0.2 \mathrm{~g}$ of pretreated biomass $10 \mathrm{~mL}$ of citrate buffer solution ( $\mathrm{pH} 4.8$ and $50 \mathrm{mM}$ ) added with $0.1 \mathrm{~g}$ of cellulase (Cellulysin, Trichoderma viride, Merck). The hydrolysis process was carried out at $50{ }^{\circ} \mathrm{C}$ for 48 hours at $100 \mathrm{rpm}$ in an incubating shaker (YLHDER, LM-400D). After hydrolysis, the samples were centrifuged at $3500 \mathrm{rpm}$ for $15 \mathrm{~min}$. The supernatant was collected and glucose content was analysed by using High Performance Liquid Chromatography (HPLC).

\subsection{Effect of pretreatment temperature and duration on sugar production}

The DES with the best performance was selected to carry out this study. The pretreatment duration and solid loading was fixed at $3 \mathrm{hr}$ and $5 \%$ when the temperature was varied from $90^{\circ} \mathrm{C}, 110^{\circ} \mathrm{C}$ and $130{ }^{\circ} \mathrm{C}$. To examine the effect of duration, DES pretreatment was carried out for 1, 3 and $5 \mathrm{hr}$ at $5 \%$ solid loading and the most suitable temperature.

\subsection{Analytical method}

\subsubsection{3, 5 Dinitrosalicyclic Acid (DNS) analysis}

DNS reagent with $1 \%(\mathrm{w} / \mathrm{v}) \mathrm{NaOH}$ (Merck), 1\% (w/v) 3,5-dinitrosalicyclic acid (DNS, Merck), $0.2 \%(\mathrm{w} / \mathrm{v})$ phenol (Merck) and 1\% (w/v) of sodium metabisulfite (Merck) was prepared. During the analysis, $3 \mathrm{~mL}$ of DNS solution was then added into the test tube which contain $1.5 \mathrm{~mL}$ of sample. The mixture was boiled for $5 \mathrm{~min}$, placed in a cold water bath before $1 \mathrm{~mL}$ of $40 \%$ Rochelle salt (Potassium sodium tartrate tetrahydrate, Merck) solution was added. $0.2 \mathrm{~mL}$ of reaction mixture was then diluted by adding $2.5 \mathrm{~mL}$ of ultrapure water and the absorbance of the solution was measured at $540 \mathrm{~nm}$ by UV-Vis spectrophotometer (Genesys10, Thermofischer Scientific). The reducing sugars 
concentration in the sample solution was determined from the standard curve constructed by using glucose solution.

\subsubsection{High Performance Liquid Chromatography (HPLC)}

Glucose concentration was examined by using HPLC (LC1220, Agilent Technology USA). Deionized water was used as the mobile phase of HPLC with flowrate of $0.6 \mathrm{ml} / \mathrm{min}$. The Agilent $\mathrm{H}+$ column was used while column temperature is fixed at $65{ }^{\circ} \mathrm{C}$. Before conducting HPLC analysis, the sample is filtered using Nylon filter with particle size of 0.2 $\mu \mathrm{m}$. Glucose concentration was determined from a calibration curve in the range of $1 \mathrm{~g} / \mathrm{L}$ to $30 \mathrm{~g} / \mathrm{L}$ glucose.

\subsubsection{Scanning Electron Microscopy (SEM) analysis}

The morphology of both untreated and DES-pretreated sago waste was observed with scanning electron microscope (Quanta 200 FESEM, FEI, USA). The images were acquired under $10 \mathrm{kV}$ acceleration voltage and magnification of $1600 \mathrm{X}$.

\subsection{Statistical analysis}

All the experiments were performed in duplicate. The significance of the results were identified by using 2-way Analysis of Variance (ANOVA) method at 95\% confidence level.

\section{Results and discussion}

\subsection{Selection of suitable DES to pretreat sago waste}

\subsubsection{Structural analysis - Scanning Electron Microscopy (SEM) Analysis}

Fig. 1. shows the SEM images of untreated and pretreated sago waste by using different DES. From the SEM analysis, all the pretreatments were successfully carried out to make the samples more susceptible for sugar extraction via enzymatic hydrolysis. Raw and untreated sago hampas as shown in Fig. 1.(a) was less porous and its structure was less disrupted. The fibers in untreated sago waste were aligned in higher degree of order as compared to its pretreated counterpart. After pretreatment, the rigid and smooth surface was disrupted and the sago waste has demonstrated higher porosity as depicted in Fig. 1.(b), (c) and (d). The disruption is crucial to make biomass more susceptible to enzymatic attack in hydrolysis process, therefore resulting higher yield of sugar. However, the morphology of ChCl-Glycerol pretreated sago waste consists of a large portion of smooth surface which indicates the fibers are still intact, thus making the enzymes less accessible to cellulose. 


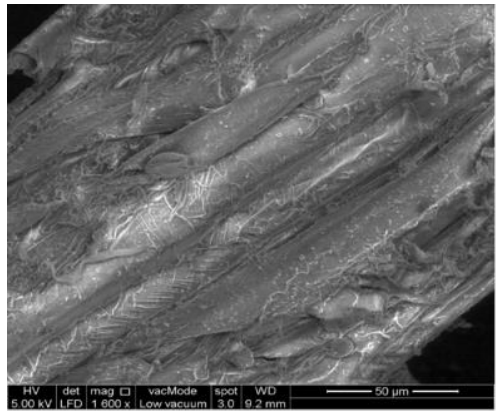

(a)

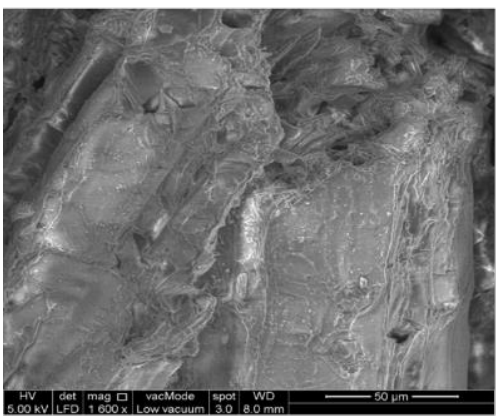

(c)

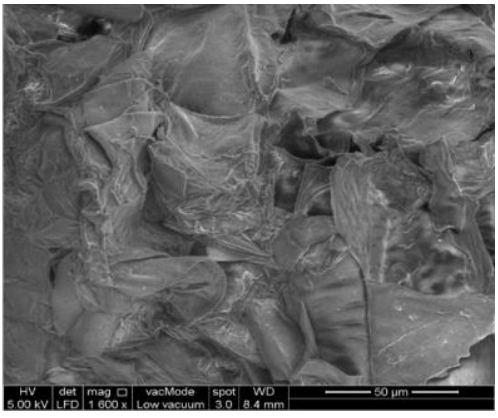

(b)

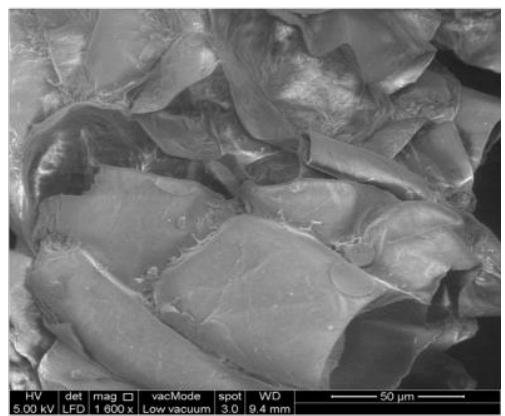

(d)

Fig. 1. SEM images of (a) untreated sago waste, (b) ChCl-Urea pretreated sago waste, (c) ChCl-CA pretreated sago waste, (d) ChCl-Glycerol pretreated sago waste at 1600x magnification.

In general, all pretreatment methods has increase the porosity of biomass and some DES might shows the ability in lignin removal [11]. A few studies have reported similar results whereby the morphology of biomass became more porous, less crystalline and more vulnerable to enzymatic hydrolysis after using ionic liquid pretreatment which has similar characteristic with DES [12]. The result from SEM analysis is aligned with biomass pretreated by using other reagents such as $\mathrm{NaOH}, \mathrm{HCl}$ and ionic liquids which concluded that alteration of biomass structure occurs in favor to microbial attack and enzymatic hydrolysis [12].

\subsubsection{Enzymatic hydrolysis of DES-pretreated sago waste}

Sago wastes was subjected to pretreatment with different types of DES at $110{ }^{\circ} \mathrm{C}, 3 \mathrm{hr}$ and $5 \%$ solid loading before enzymatic hydrolysis. The performance of these DES pretreatments was analysed and justified based on yield of reducing sugar obtained from enzymatic hydrolysis and sugar loss during pretreatment besides examining the structural characteristics as outlined in Section 3.1.1. 


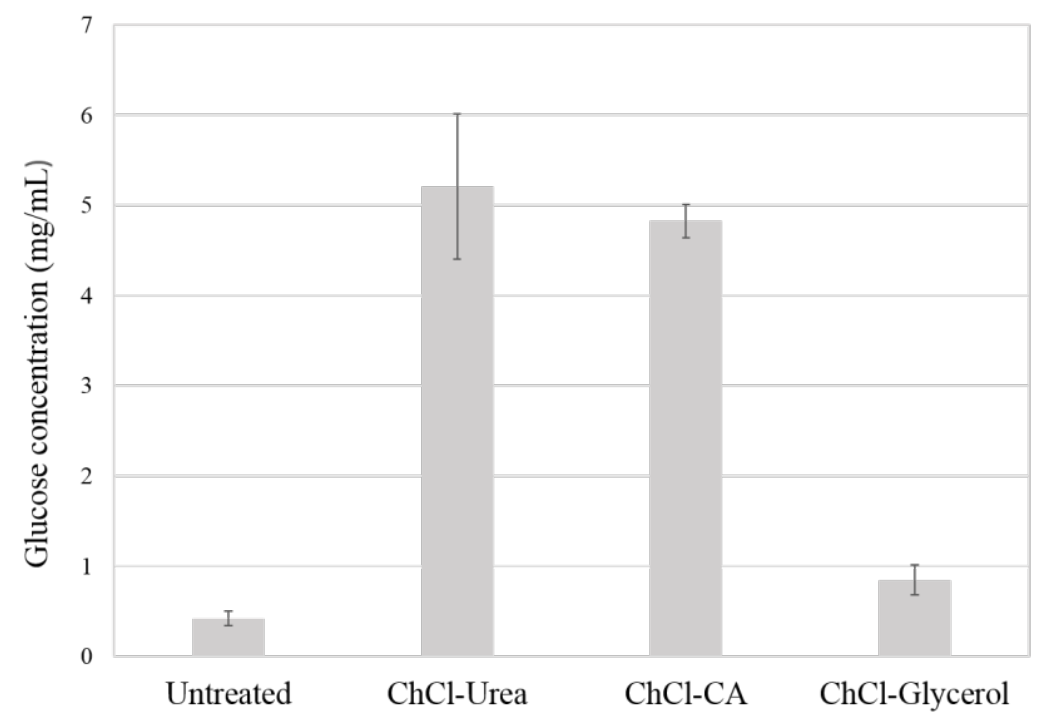

Fig. 2. Glucose yield from enzymatic hydrolysis of sago wastes pretreated by different types of DES

From Fig.2., ChCl-Urea pretreated sago waste gave the highest amount of glucose yield at $5.2 \mathrm{mg} / \mathrm{ml}$ (which is $0.26 \mathrm{~g} / \mathrm{g}$ of pretreated biomass) compared to ChCl-Glycerol and ChClCA pretreated biomass. It was found that sago waste pretreated by ChCl-Glycerol yielding a similar glucose yield with the untreated sample which renders this DES the least effective in pretreatment of sago waste. From 2-way ANOVA analysis, the glucose yield obtained from different DES pretreatment was significant $(\mathrm{F}=36.56)$ whereas the batch-to-batch difference $(\mathrm{F}=0.22)$ was insignificant under $95 \%$ confidence level.

Based on the nature of each DES, urea is slightly alkaline with $\mathrm{pH}$ approximately 9 whereas both glycerol and citric acid are acidic with $\mathrm{pH}$ at $6.3-6.9$ and 3.2 respectively. From the results obtained, it can be concluded that sugar yield increases when wither acidic or alkaline reagent was used in pretreatment. ChCl-Urea might act just like the reagent in conventional alkaline pretreatment to break down lignin and facilitates the access of enzymes to biomass. On the other hand, ChCl-glycerol, which is having neutral $\mathrm{pH}$, yielded the lowest amount of glucose with $0.9 \mathrm{mg} / \mathrm{ml}$ (which is $0.04 \mathrm{~g} / \mathrm{g}$ of pretreated biomass). This finding aligns with a research done which stated that the maximal enzymatic glucose and xylose yields from the biomass, wheat straw fraction were noticed at the most extreme $\mathrm{pH}$ values ( $\mathrm{pH} 1$ and $\mathrm{pH} 13)$ after pretreatment [13]. This trend can be further explained as the surface response models showed significant interactions between pretreatment temperature and $\mathrm{pH}$ on glucose and xylose [13]. Acidic pretreatment was favorable at high petreatment temperature compared to alkaline pretreatment, which is not as temperature dependent. From research, alkaline pretreatment generally proven to have better glucose yield even at lower pretreatment $\left(110^{\circ} \mathrm{C}\right)$ temperature as compared to acidic pretreatment [13]. Therefore, the results obtained from Fig.2. were found to be agreeable when slightly lower yield of reducing sugar from was obtained from sago waste pretreated by acid-based DES than alkali-based DES.

\subsubsection{Reducing sugar loss during pretreatment}

To further confirm the suitability of using DES-pretreated sago waste for glucose production via enzymatic hydrolysis, reducing sugar loss during DES pretreatment was examined and the results are tabulated in Table 1. As such, DES was collected after 
pretreatment and the reducing sugar content in DES solution was analysed. DES pretreatment which contributes to a higher sugar loss is unfavourable in this case.

Table 1. Reducing sugar loss during pretreatment

\begin{tabular}{|c|c|}
\hline Types of DES & Reducing sugar concentration in DES (mg/mL) \\
\hline ChCl-Urea & $0.9 \pm 0.14$ \\
\hline ChCl-Glycerol & $2.0 \pm 0.28$ \\
\hline ChCl-CA & $0.8 \pm 0.14$ \\
\hline
\end{tabular}

From Table 1, during ChCl-Glycerol pretreatment, the amount of reducing sugar loss was recorded to be twice as much as $\mathrm{ChCl}$-Urea and ChCl-CA pretreatments. Similar to strong acid and some ionic liquids, DES might also be able to depolymerise polysaccharides to compounds with low molecular weight [14], and this causes reducing sugar loss was observed. Based on Table 1, ChCl-Glycerol can be said to have the highest depolymerisation power followed by $\mathrm{ChCl}-\mathrm{Urea}$ and lastly, $\mathrm{ChCl}-\mathrm{CA}$. The mechanism of ChCl-glycerol in depolymerisation of biomass remained unknown and worth investigating. Since reducing sugar loss is unfavourable as it is more beneficial to retain the sugar within the pretreated biomass for subsequent enzymatic hydrolysis, $\mathrm{ChCl}$-urea which produced the highest amount of sugar during enzymatic hydrolysis and shows low reducing sugar loss was chosen in this study. This was further supported by the structural analysis whereby $\mathrm{ChCl}$-urea pretreated sago waste has a more porous and disrupted structure. Based on the DES chosen, a preliminary study on suitable temperature and duration for pretreatment of sago waste was then conducted.

\subsection{Effect of temperature on DES pretreatment}




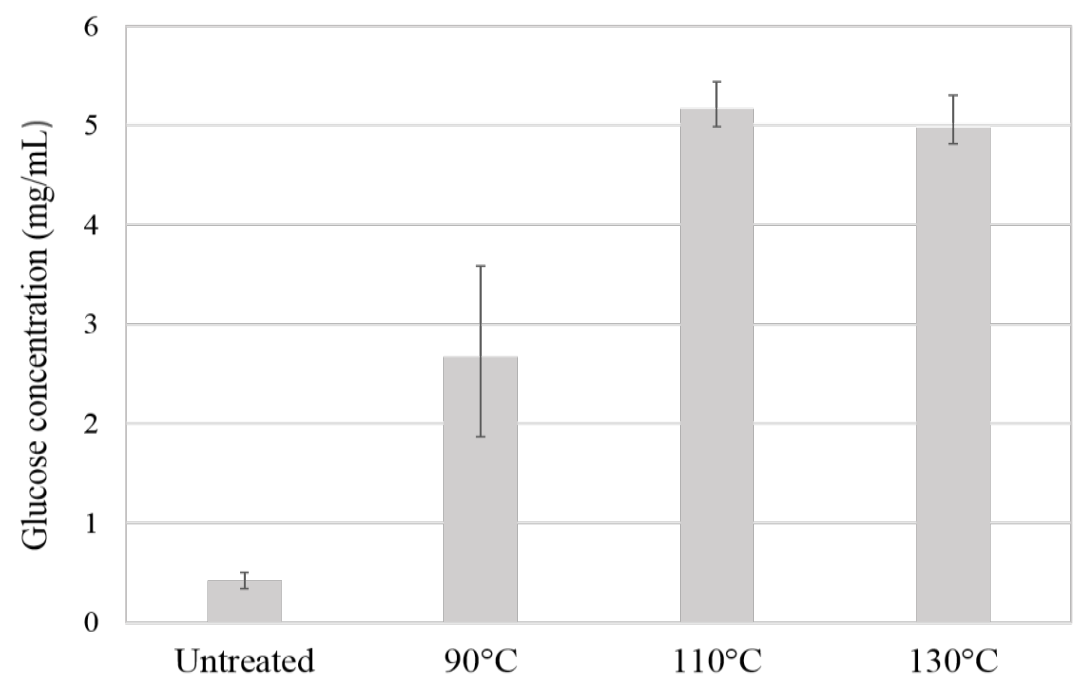

Fig. 3. Glucose concentration at $3 \mathrm{hr}$ under different temperature

Based on Fig. 3., glucose concentration increases from $90^{\circ} \mathrm{C}$ to $110^{\circ} \mathrm{C}$ and reduced slightly at $130^{\circ} \mathrm{C}$. The result shows the optimum temperature would be at $110^{\circ} \mathrm{C}$ with $5.2 \mathrm{mg} / \mathrm{ml}$ (which is $0.26 \mathrm{~g} / \mathrm{g}$ of pretreated biomass) of glucose yield. The trend is aligned to a DES pretreatment study using $\mathrm{ChCl}$-lactic acid to pretreat corncob with varying pretreatment temperature as efficiency of glucose extraction started to decline at certain temperature. The losses of polysaccharides could be due to the balanced effects to improve digestability of hydrolysis [9]. Also, high temperature may contribute to degradation of biomass samples, thus lead to sugar loss. This is further explained by higher pretreatment temperature increases the deconstruction of biomass components and improves amount of sugar produced [10]. Moreoever, at higher temperature, increment in pore size of biomass along with reduction of lignin is expected to happen within the biomass [10].

\subsection{Effect of duration on DES pretreatment}

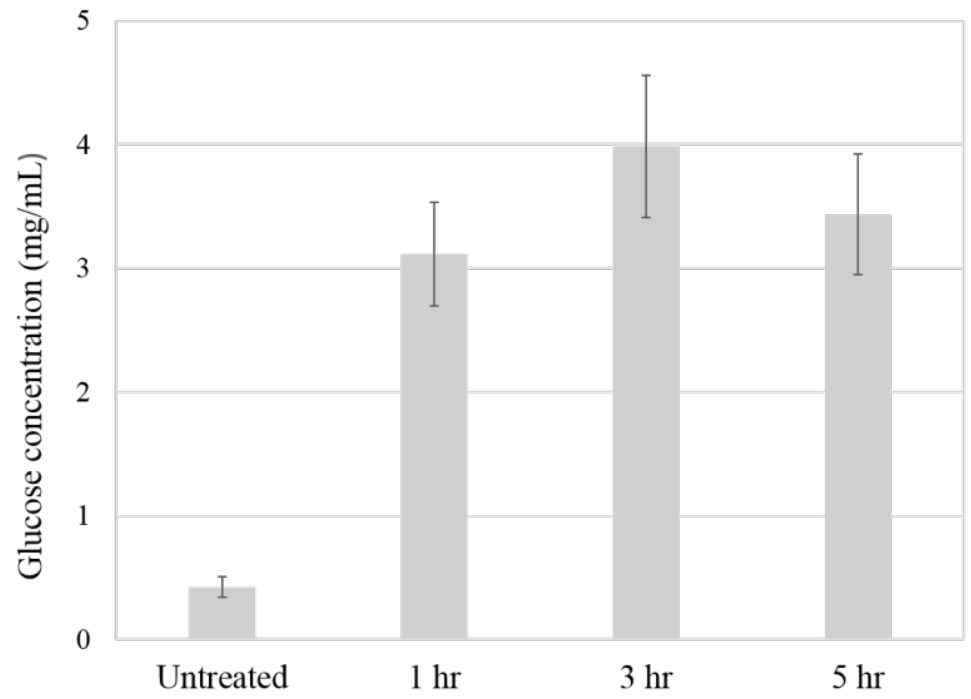


Fig. 4. Glucose concentration at $110{ }^{\circ} \mathrm{C}$ under different duration

Based on Fig. 4., it can be observed that glucose concentration of $4.0 \mathrm{mg} / \mathrm{mL}$ (which is 0.2 $\mathrm{g} / \mathrm{g}$ of pretreated biomass) was the highest when pretreatment was applied for $3 \mathrm{hr}$. This was followed by $5 \mathrm{hr}$ and $1 \mathrm{hr}$ with glucose concentration of $3.4 \mathrm{mg} / \mathrm{ml}$ and $3.1 \mathrm{mg} / \mathrm{ml}$ respectively. Glucose yield was seen declining at the $5^{\text {th }} \mathrm{hr}$. The sugar loss that might happen at a more profound rate and the degradation of biomass are two possible factors that would cause a decrease in glucose yield when pretreatment duration is extended to $5 \mathrm{hr}$. This finding agrees well with recent research in which glucose yield increases significantly with pretreatment duration and gives better hydrolysis efficiency until the approximately the $3^{\text {rd }}$ hour before a consistent glucose yield was obtained thereafter [9]. The experiment done by using corncob under different pretreatment duration found that better interaction between biomass and DES-based solvent was expected under prolonged duration [9]. From Fig.4., the most suitable pretreatment duration is $3 \mathrm{hr}$ because the highest amount of sugar yield was obtained.

This study highlights the use of DES as potential green solvents for pretreatment process. At current stage, limited study is available as reference on the application of DES in pretreatment of lignocellulosic biomass. This study could serves as a reference on how DES pretreatment affects the sugar production and structure from starchy biomass. The development in this field would promote and lead to application of eco-friendly pretreatment technology in the future.

\section{Conclusion}

This preliminary study has demonstrated the potential application of DES in pretreating starchy biomass. DES pretreatment was able to produce biomass with higher porosity and lower crystallinity which is beneficial for the subsequent enzymatic hydrolysis process. It was found that alkaline or acidic-based DES are more suitable in pretreating sago waste as compared to DES with neutral $\mathrm{pH}$. This is because a higher glucose yield were obtained from enzymatic hydrolysis of sago waste pretreated by alkaline and acidic-based DES. Reducing sugar loss during pretreatment was found to be lower when ChCl-urea (alkalinebased DES) and ChCl-CA (acidic-based DES) were ussed. ChCl-Urea was chosen to pretreat sago waste in this study as it demonstrates a high glucose yield from enzymatic hydrolysis with low reducing sugar loss during pretreatment besides the favorable structural changes. The best operating condition for sugar production from $\mathrm{ChCl}$-Urea pretreated sago waste via enzymatic hydrolysis is at $110^{\circ} \mathrm{C}$ and $3 \mathrm{hr}$. Further investigation on the compositional analysis of pretreated biomass as well as the optimization of pretreatment condition could be conducted to further elucidate the effectiveness of the pretreatment process.

\section{References}

1. F. Isikgor, C. R. Becer, Polym. Chem. 6, 4497 - 4559 (2015)

2. S. S. da Silva, A. K. Chandel, S. R. Wickramasinghe, J. M. G. Domínguez, J. Biomed. Biotechnol. 2012, 1-2 (2012)

3. A. Limayem, S. C. Ricke, Prog. Energy Combust. Sci. 38, 449-467 (2012)

4. A. K. Kumar, S. Sharma, Bioresour. Bioprocess. 4, 7 (2017)

5. Y. P. Mbous, M. Hayyan, A. Hayyan, W. F. Wong, M. A. Hashim, C. Y. Looi, Biotechnol. Adv. 35, 105-134 (2017)

6. K. Ellinor B. Heggset, Kristin.S, Biomass Bioenergy 93, 194-200 (2016) 
7. A.K. Kumar, B.S. Parikh, M. Pravakar, Environ. Sci. Pollut. Res. 23, 9265 - 9275 (2016)

8. A. Procentese, E. Johnson, V. Orr, A. Garruto Campanile, J.A. Wood, A. Marzocchella, L. Rehmann, Bioresour. Technol. 192, 31 - 36 (2015)

9. C.W. Zhang, S.Q. Xia, P.S. Ma, Bioresour. Technol. 219, 1-5 (2016)

10. A. N. Gunny, D. Arbain, E. M. Nashef, P. Jamal, Bioresour. Technol. 181, 297-302, (2015)

11. N. Nor, W. Mustapha, O. Hassan, Procedia Chem. 18, 147-154 (2016)

12. L. W. Yoon, T. N. Ang, G. C. Ngoh, A. S. M. Chua, Biomass Bioenergy 36, 160-169 (2012)

13. M. Pedersen, K. S. Johansen, A. S. Meyer, Biotechnol. Biofuels 4, 11 (2011)

14. D. Di Marino, D. Stöckmann, S. Kriescher, S. Stiefel, M. Wessling, Green Chem. 18, 6021-6028 (2016) 